
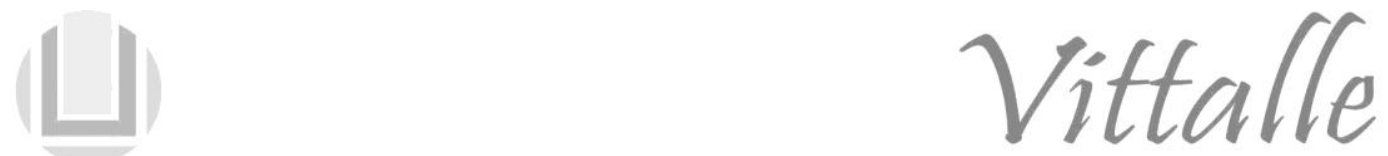

\title{
A infecção pelo gênero Plasmodium: epidemiologia, profilaxia e controle no Brasil
}

\author{
Andréia Patrícia Gomes ${ }^{\mathrm{a}, *}$, Rodrigo Roger Vitorino ${ }^{\mathrm{b}}$, Thainara Aparecida Mendes ${ }^{\mathrm{c}}$, \\ Sandra de Oliveira Pereira ${ }^{a}$, Paulo Sérgio Balbino Miguel ${ }^{a}$, Luciene Muniz Braga ${ }^{c}$, \\ Tiago Ricardo Moreira ${ }^{a}$, Luiz Alberto Santana ${ }^{a}$
}

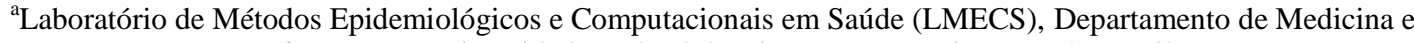
Enfermagem, Universidade Federal de Viçosa (UFV), Viçosa, MG, Brasil.

${ }^{\mathrm{b}}$ Centro Universitário Serra dos Órgãos (UNIFESO), Teresópolis, RJ, Brasil

${ }^{c}$ Departamento de Medicina e Enfermagem, Universidade Federal de Viçosa (UFV), Viçosa, MG, Brasil.

Histórico do Artigo

Recebido em

09/11/2017

Aceito em 05/03/2018

Palavras-chave:

Malária;

Epidemiologia;

Prevenção e controle.

Key words:

Malaria; Epidemiology;

Prevention and control.

\begin{abstract}
RESUMO
A malária, doença provocada por espécies de protozoários do gênero Plasmodium, é a parasitose de maior relevância no mundo. No Brasil, há uma maior incidência de infecções provocadas pelo Plasmodium vivax, destacando-se que a enfermidade por Plasmodium falciparum é classicamente a mais grave. A abordagem da malária - em termos individuais e de saúde pública - depende de um conhecimento dos principais aspectos da moléstia. Diante disso, realizou-se uma revisão da literatura com o objetivo de apresentar os aspectos epidemiológicos, profiláticos e de controle da malária no Brasil. A revisão contemplou consulta à PubMed (U.S. National Library of Medicine), SciELO (Scientific Eletronic Library Online) e Lilacs (Literatura Latino-Americana e do Caribe em Ciências da Saúde). Os resultados mostraram que a malária é um importante problema de saúde pública e uma das principais causas de morte por doenças infecciosas, resultando em mais de 300 milhões de casos e cerca de um milhão de óbitos por ano. No Brasil, a ocorrência de malária é concentrada na área da Amazônia Legal, compreendendo os seguintes estados: Acre, Amazonas, Amapá, Rondônia, Roraima, Pará,Tocantins, Maranhão e Mato Grosso. A despeito dos avanços no tratamento da malária, destaca-se a grande relevância das ações de profilaxia e de controle da doença, as quais têm repercussões - positivas - em termos individuais e coletivos.
\end{abstract}

Plasmodium genus infection: epidemiology, prevention and control in Brazil

\section{ABSTRACT}

Malaria is the most important parasite disease in the world. Caused by a protozoan species of the genus Plasmodium, in Brazil the highest incidence of infections are by Plasmodium vivax; however the Plasmodium falciparum disease is normally the most serious. The approach to malaria in terms of individual and public health depends on an understanding of the major aspect of epidemiology. In view of this, we performed a literature review about the epidemiological, prophylactic and control aspects of malaria in Brazil. PubMed (U. S. National Library of Medicine), SciELO (Scientific Electronic Library Online) and Lilacs (Latin American and Caribbean Health Sciences) were consulted. Results showed that malaria is an important public health problem worldwide and a leading cause of death from infectious diseases, resulting in more than 300 million cases and nearly one million deaths per year. In Brazil, the incidence of malaria is concentrated in the area of the Amazon, including the following states: Acre, Amazonas, Amapá, Rondônia, Roraima, Pará, Tocantins, Maranhão and Mato Grosso. Despite of the advances in the treatment of malaria, highlights the great importance of the actions of prevention and control of the disease, which have positive impact at the individual and collective level.

\section{Introdução}

A malária é uma protozoose febril causada, usualmente, por quatro espécies de protozoários do gênero Plasmodium: Plasmodium falciparum, Plasmodium vivax, Plasmodium malariae e Plasmodium ovale, ainda que, mais recentemente, infecções por

\footnotetext{
*Autor correspondente: andreiapgomes@ gmail.com (Gomes A. P.)
} 
Plasmodium knowlesi venham sendo descritas $(1,2)$. A etiologia da maior parte dos casos é dada por $P$. vixax (no Brasil, cerca de 90\% deles) (3). A forma grave e letal da doença é resultado, habitualmente, de infecção por $P$. falciparum. A infecção por $P$. malariae representa uma minoria dos casos e $P$. ovale está presente somente no continente Africano $(3,4)$.

A malária se apresenta, em muitos casos, como doença de difícil identificação, sobretudo para profissionais de saúde que não residem em áreas endêmicas. Deve-se investigar a hipótese diagnóstica de malária em pacientes com história epidemiológica compatível (estada em área endêmica) ou naqueles que tenham recebido transfusão de sangue e hemoderivados e que apresentem febre alta contínua ou em paroxismos (5). A confirmação do diagnóstico pode ser feita por meio de distensão sanguínea ou gota espessa coradas pelo Giemsa (3).

Em alguns casos, especialmente na infecção por P. falciparum, o estabelecimento do diagnóstico e a instituição precoce da terapêutica específica podem representar a única chance de sobrevida do indivíduo acometido. Destaca-se, igualmente, que a profilaxia e o controle - baseados, especialmente, nas seguintes medidas: detecção e tratamento (imediato) de novos casos; investigação dos casos; orientação à população quanto à doença, uso de repelentes, mosquiteiros, roupas protetoras, telas em portas e janelas; emprego da quimioprofilaxia - são de vital importância para a redução dos índices de malária.

Com base nessas breves considerações, o objetivo do presente artigo é apresentar uma revisão dos aspectos epidemiológicos, profiláticos e de controle da malária no Brasil.

\section{Métodos}

Para a revisão da literatura, foram consultadas as fontes PubMed (U. S. National Library of Medicine), SciELO (Scientific Eletronic Library Online), e Lilacs (Literatura Latino-Americana e do Caribe em Ciências da Saúde) por representarem bases de dados informatizadas de acesso gratuito para pesquisa de artigos científicos e consideradas fontes primárias de informação. Os termos pesquisados foram definidos com base nos Descritores em Ciências da Saúde (Decs), a partir de duas estratégias de busca: (a) malária + epidemiologia + Brasil; (b) malária + controle + Brasil. A busca resultou em 931 citações (Quadro 1).

Quadro 1: Número de citações obtidas na pesquisa bibliográfica.

\begin{tabular}{|l|l|l|l|}
\hline Estratégia de busca* & \multicolumn{2}{l|}{ Base consultada } & \multicolumn{1}{l|}{ SciELO } \\
\hline Estratégia 1: malária + epidemiologia + Brasil & 37 & 262 & 50 \\
\hline Estratégia 2: malária + controle + Brasil & 240 & 252 & 90 \\
\hline
\end{tabular}

* Data limite para a busca: 31/01/2018.

**Para pesquisa na base de dados PUBMED - empregando termos em língua inglesa.

A primeira seleção foi feita por meio da leitura criteriosa do título e do resumo online, selecionando-se (i) os estudos originais e (ii) as revisões bibliográficas disponíveis na íntegra, publicados nas línguas espanhola, inglesa e portuguesa; foram descartados os textos não adequados ao objetivo do artigo e as duplicidades encontradas. Posteriormente, os textos escolhidos nessa primeira análise foram lidos na íntegra e avaliados em relação à proposta da revisão, excluindo-se - ao final - 880 citações. Com efeito, do total de citações originalmente obtidas, foram finalmente selecionados 51 textos, centrados na epidemiologia e no controle da malária no Brasil. Além do emprego dos artigos escolhidos, também foram consultados livros-texto de epidemiologia, 
infectologia e medicina interna - e outras fontes bibliográficas de conhecimento prévio dos autores - como parte integrante do levantamento bibliográfico.

A partir da leitura dos manuscritos selecionados, procedeu-se a organização das informações obtidas, as quais serão apresentadas nas três seções a seguir EPIDEMIOLOGIA, PROFILAXIA E CONTROLE - de acordo com a seguinte lógica: a) Distribuição geográfica e impacto epidemiológico, b) Dinâmica de transmissão da moléstia, c) Emergência e reemergência da malária, d) Malária e infecção pelo HIV, e) Malária resistente e multirresistente no Brasil, f) Proteção individual, g) Quimioprofilaxia, h) Controle vetorial e i) Vacinação para malária.

\section{Epidemiologia}

\subsection{Distribuição geográfica e impacto epidemiológico}

A malária é um grave problema de saúde pública no mundo e uma das principais causas de morte por doenças infecciosas, devido a sua alta incidência e às consequências fisiopatológicas para as pessoas acometidas, resultando em mais de 300 milhões de casos e cerca de um milhão de desenlaces fatais por ano (6). Tal condição mórbida coloca sob risco cerca de $40 \%$ da população mundial, em mais de 109 países (6). Sua maior incidência ocorre no continente africano, principalmente ao sul do deserto do Saara $(7,8)$.

No continente americano, o Brasil concentrava o maior número de casos de malária até o ano de 2015 (ano que registrou 143.161 casos). No ano de 2016, foi ultrapassado pela Venezuela, que registrou 242.561 casos comparado aos 137.996 registros em 2015. Em termos de incidência anual, o Brasil apresentou a quinta maior incidência de malária na américa em 2016, seguido pela Guiana, Venezuela, Peru e Colômbia (9).

De janeiro a outubro de 2017, foram notificados 154.566 casos de malária no Brasil, representando um aumento de 47\% em relação ao ano de 2016 (105.057 casos notificados) (10) - destacando-se que a área endêmica concentra-se na Amazônia Legal, que engloba os Estados do Acre, Amapá, Amazonas, Maranhão, Mato Grosso, Pará, Rondônia, Roraima e Tocantins (11). Entretanto, a Amazônia brasileira não é homogênea no que se refere à distribuição da malária, havendo áreas de alta, média e baixa transmissão, bem como regiões nas quais a moléstia não é transmitida (12). Segundo o Ministério da Saúde, $P$. vivax é a espécie responsável por cerca de $90 \%$ dos casos (8). No entanto, a forma grave e letal da doença é causada pela infecção por $P$. falciparum, a qual tem apresentado redução importante nos últimos anos (3).

Os fatores combinados - na região amazônica - que favorecem a transmissão da doença e dificultam as medidas de prevenção e de controle, incluem a) o calor, a umidade e as coleções hídricas abundantes - elementos que propiciam condições para o desenvolvimento do mosquito vetor, b) os aspectos antrópicos relacionadas à habitação (casas destituídas de paredes, impossibilitando a aplicação de inseticidas residuais), c) as dificuldades para plena operacionalização de programas de borrifação de inseticidas e d) a dispersão demográfica da área (13).

Os estados de São Paulo, Minas Gerais, Rio de Janeiro e Espírito Santo são os que apresentam maior número de notificações de malária fora da região amazônica. Quando se observa os casos autóctones, os estados do Rio de Janeiro e Espírito Santo são os que apresentam maior número de casos notificados, sendo as regiões serranas dos dois estados os locais que apresentam maior risco de transmissão da doença. De janeiro a outubro de 2017, foram notificados 499 casos de malária extra-amazônica (10). Apesar de representar apenas 3\% do total de casos notificados no Brasil nesse período, esse 
número chama atenção uma vez que a letalidade da malária na região extra-amazônica é cerca de 80 vezes maior do que na Amazônia (14).

\subsection{Dinâmica de transmissão da moléstia}

Os mosquitos vetores do Plasmodium spp pertencem ao gênero Anopheles (classe Insecta, ordem Diptera, família Culicidae) (15). Apenas as fêmeas do mosquito têm hábito hematofágico, possuindo, portanto, capacidade de transmissão (os machos alimentam-se da seiva de vegetais). Os mosquitos do gênero Anopheles se infectam ao picar o Homo sapiens sapiens doente (preferencialmente no crepúsculo), acreditando-se que, excepcionalmente, alguns chimpanzés na África e outros símios na América do Sul possam servir de fonte de infecção para $P$. malariae (16).

No Brasil, as principais espécies transmissoras do gênero pertencem a dois subgêneros: Nyssorrynchus e Kerteszia. O Anopheles (Nyssorrynchus) darlingi é o principal vetor no país, na medida em que seu comportamento é extremamente antropofílico e, dentre as demais espécies, é aquela que mais se situa no peridomicílio ${ }^{4}$. Na faixa litorânea - estendendo-se do Amapá ao norte de São Paulo - descreve-se o Anopheles (Nyssorrynchus) aquasalis com grande relevância epidemiológica (destacando-se, em termos biológicos, sua proliferação preferencialmente em água salobra). Anopheles (Kerteszia) cruzi e Anopheles (Kerteszia) aquasalis distribuem-se igualmente pelo litoral - Sul de São Paulo ao Norte do Rio Grande do Sul -, representando um problema em termos de controle, por seu hábito de constituir criadouros na água acumulada sobre a base das folhas de bromélias (17).

Além da malária natural, descreve-se a transmissão da doença a partir das hemotransfusões (18), dos acidentes com material perfurocortante em laboratórios ou na atividade profissional de saúde, da introdução de vetores infectados pelo agente trazidos por meios de transporte, como por exemplo, o avião ("malária do aeroporto") (19).

Nos anofelinos, a fêmea do mosquito do gênero Anopheles ingere os gametócitos quando pica o homem doente. Tais formas, no tubo digestivo, transformam-se em gametas. Os gametócitos dão origem ao oocineto (zigoto) por meio da reprodução sexuada. Essas formas penetram a parede do tubo digestivo do hospedeiro invertebrado, transformando-se em oocistos que, por aumento progressivo de tamanho e divisão (esporogonia), produzem numerosos esporozoítas -, que ganham a cavidade celômica e atingem as glândulas salivares - sendo inoculados pela saliva do inseto durante a picada. Tal ciclo tem duração, em média, de 7 a 21 dias (15).

\subsection{Emergência e reemergência da malária}

A despeito dos enormes esforços para seu controle e erradicação, a doença está ressurgindo em muitas regiões tropicais. Atribui-se essa expansão da enfermidade à ação antrópica desordenada (20) - como o desmatamento, que altera o habitat do agente transmissor - e aos crescentes problemas de resistência do Plasmodium aos fármacos e dos vetores aos inseticidas (4). Um dos problemas relacionados aos fármacos está associado aos polimorfismos no citocromo P-450 (CYP2D6), que aumentam o risco de recorrência da malária causada por $P$. vivax, após a terapia combinada com cloroquinaprimaquina (21).

Surtos também podem surgir em decorrência da imigração de pessoas não imunes à doença para regiões endêmicas ou de pessoas de áreas endêmicas para regiões que até então eram livres de malária. Evidências apontam para a reemergência da doença em regiões como o planalto do nordeste da Tanzânia e o oeste de Nova Guiné (22). 
No Brasil, é importante salientar que a região extra-amazônica abriga várias espécies de mosquitos com potencial para transmitir malária. Com as mudanças na paisagem e no clima do país, é possível que espécies antes inofensivas passem a transmitir o Plasmodium. Na região extra-amazônica, a malária tornou-se um problema que afeta principalmente subpopulações isoladas com certas características sociais (por exemplo, tipos de habitação) ou que desempenham específicas atividades profissionais. Portanto, a educação adequada dos indivíduos em risco e profissionais de saúde é necessária. Além disso, meios de diagnóstico rápido devem ser implementados nessas regiões para evitar eventos adversos graves ou mortes por malária (23).

Com relação à malária causada por Plasmodium knowlesi, um em cada dez pacientes com essa condição mórbida desenvolve complicações. A mesma não estava sendo diagnosticada por exames microscópicos devido à semelhança entre o $P$. knowlesi e os $P$. malariae e $P$. falciparum. O diagnóstico pode ser feito mediante reação de polimerase em cadeia (PCR), a partir da identificação de primers específicos; entretanto o acesso aos procedimentos de sequenciamento de DNA é limitado em áreas endêmicas. Para uma correta avaliação da incidência, natureza e características clínicas da malária por esse protista emergente, seria necessário notificar os casos de infecção por P. knowlesi em viajantes, coletando os dados clínicos e epidemiológicos $(1,2)$.

\subsection{Malária e infecção pelo HIV}

A ocorrência de malária em indivíduo previamente infectado pelo vírus da imunodeficiência humana (HIV) causa impacto negativo para ambas as moléstias. As populações infectadas pelo HIV apresentam importantes particularidades no contexto da exposição ao Plasmodium em comparação à população geral, exibindo um maior risco de: a) adquirir malária (24); b) desenvolver sua forma grave $(25,26)$; c) apresentar sintomas mais acentuados (27); e d) de evoluir com fracasso terapêutico (28).

A malária e a infecção pelo HIV induzem respostas imunocelulares semelhantes, as quais proporcionam um microambiente favorável à propagação do vírus entre os linfócitos T CD4+ produzindo, ato contínuo, rápida replicação viral, acentuado declínio numérico das células CD4+ e agravamento de ambas as moléstias $(29,30)$.

Para a proteção dos pacientes infectados pelo HIV que residem em áreas endêmicas de malária são necessárias algumas intervenções profiláticas - tais como o uso de mosquiteiros tratados com inseticida (31) - as quais serão detalhadas adiante.

\subsection{Malária resistente e multirresistente no Brasil}

A taxa em que a resistência a drogas antimaláricas está emergindo e sua disseminação está superando o desenvolvimento de novas drogas. Isso tem se traduzido em maior carga da doença e também maior gravidade dos casos $(32,33)$. Dentre os fatores que contribuem para a resistência às drogas antimaláricas, pode-se citar o uso intensificado de fármacos, a seleção do parasita e a resistência cruzada aos medicamentos. No Brasil, cerca de $90 \%$ dos pacientes infectados por $P$. falciparum não respondem à cloroquina (34). A resistência das cepas de $P$. falciparum a esse fármaco, na região amazônica, é de quase $100 \%$. Um tratamento amplamente utilizado inclui a associação entre sulfadoxina-pirimetamina; no entanto, a resistência a tal esquema já tem sido descrita. Embora a distribuição dessas cepas resistentes ainda não seja bem conhecida, sabe-se que sulfadoxina-pirimetamina não é um tratamento eficaz na região amazônica $(31,35)$.

A Organização Mundial da Saúde (OMS) recomenda que todos os países que enfrentam resistência às monoterapias convencionais para o tratamento da malária 
causada pelo $P$. falciparum utilizem terapias combinadas, preferencialmente, de medicamentos contendo derivados de artemisinina (35).

Também já foram relatados, no país, casos de malária por $P$. vivax não responsivos a cloroquina $(31,35)$. Plasmodium ovale e Plasmodium malariae são sensíveis aos atuais medicamentos antimaláricos (36).

\section{Profilaxia}

As medidas profiláticas da malária se articulam, especialmente, às medidas de proteção individual - providências tomadas na tentativa de reduzir a exposição do indivíduo ao vetor, insetos do gênero Anopheles - e a profilaxia com medicamentos indicados a pessoas não imunes que farão viagens a áreas endêmicas. Tais condutas não são totalmente efetivas e mesmo com quimioprofilaxia adequada, pode haver a transmissão e o adoecimento por malária $(37,38)$.

\subsection{Proteção individual}

Em relação à minimização do risco de contato com o vetor, é preconizada a utilização de roupas de mangas compridas, calças e meias, visando à diminuição da área corporal exposta. Entretanto, esse conselho é, muitas vezes, desprovido de cunho prático, pois as áreas de maior risco no Brasil estão localizadas na Região Amazônica, onde as condições climáticas - calor e umidade - podem ser genuínos empecilhos para a adoção de tais proposições. Uma alternativa é o uso diário de inseticidas repelentes no ambiente domiciliar e, ainda, dispositivos antimosquitos de liberação lenta, já que o vetor tem hábito preferencialmente noturno e intra ou peridomiciliar (37). Em relação à transmissão no domicílio, medidas gerais de arquitetura podem ser adotadas visando à menor circulação dos vetores, destacando-se a utilização de telas nas portas e nas janelas e a construção de habitações com paredes inteiras. Existem métodos de impregnação de roupas e de mosquiteiros com substâncias do grupo das permetrinas, que são comprovadamente eficazes na diminuição da exposição, podendo ser empregados com segurança (39).

\subsection{Quimioprofilaxia}

A quimioprofilaxia - a qual consiste no uso de fármacos antimaláricos em esquemas posológicos especiais (geralmente com doses inferiores àquelas empregadas na terapêutica) - é assunto controverso devido à possibilidade de efeitos adversos, a não aderência ao esquema proposto e à resistência crescente do gênero Plasmodium ao redor do mundo. De um modo geral, tal conduta é recomendada: a) quando o risco de doença grave ou morte por $P$. falciparum for maior que os efeitos adversos dos fármacos empregados, b) se a avaliação do risco indicar alta probabilidade de adquirir malária grave e c) se a permanência do indivíduo em áreas de transmissão da malária for menor que seis meses (40). Atualmente, quatro esquemas podem ser empregados para profilaxia: doxiciclina, mefloquina, a combinação atovaquona/proguanil e cloroquina (3). Em áreas de baixa resistência do $P$. falciparum, pode-se utilizar o artemether/lumefantrina e / ou o quinino associado à doxiciclina ou à clindamicina (40).

Para a profilaxia adequada, deve-se iniciar o fármaco uma semana antes da viagem para a área endêmica, objetivando o alcance de concentrações séricas adequadas do medicamento, além da detecção de possíveis efeitos colaterais. Após saída da área de transmissão do Plasmodum, é conveniente a manutenção do fármaco por mais quatro semanas (40). 
Quadro 2: Fármacos e doses para a quimioprofilaxia da malária.

\begin{tabular}{|l|l|l|}
\hline Fármacos & Dose adulto & Duração \\
\hline Doxiciclina & $100 \mathrm{mg} / \mathrm{dia}$ & $\begin{array}{l}\text { Iniciar um dia antes da viagem e manter até } \\
\text { quatro semanas após o retorno. }\end{array}$ \\
\hline Mefloquina & $\begin{array}{l}\text { Iniciar pelo menos uma semana } \\
\text { (preferencialmente três semanas) antes da } \\
\text { viagem e manter até quatro semanas após o } \\
\text { retorno. }\end{array}$ \\
\hline Atovaquona/proguanil & $250+100 \mathrm{mg} / \mathrm{dia}$ & $\begin{array}{l}\text { Iniciar um dia antes da viagem e manter até } \\
\text { sete dias após o retorno. }\end{array}$ \\
\hline Cloroquina & $300 \mathrm{mg} / \mathrm{semana}$ & $\begin{array}{l}\text { Iniciar um dia antes da viagem e manter até } \\
\text { quatro semanas após o retorno. }\end{array}$ \\
\hline
\end{tabular}

Brasil. Ministério da Saúde. Secretaria de Vigilância em Saúde. Diretoria Técnica de Gestão. Guia para profissionais de saúde sobre prevenção da malária em viajantes / Ministério da Saúde, Secretaria de Vigilância em Saúde, Diretoria Técnica de Gestão. - Brasília: Ministério da Saúde, 2008.

A quimioprofilaxia não é absolutamente eficaz, podendo ocorrer falhas e aparecimento da doença com adiamento das manifestações clínicas. Portanto, é necessário orientar o viajante quanto aos sinais e sintomas da doença, para que possa acessar um serviço de saúde se apresentá-los (40).

\section{Controle}

O ano de 2010 marcou uma série de acordos internacionais para o controle da malária (41). No Brasil, essas ações foram direcionadas no sentido de (38):

- compromisso político com o gravíssimo problema de saúde pública representado pela malária no país, incluindo planos de desenvolvimento da Região Amazônica no controle da moléstia;

- orientação da formação de recursos humanos para o Sistema Único de Saúde (SUS) para a implementação de ações de controle;

- participação efetiva da população através da educação para a saúde.

Tais proposições visam, em última instância:

- ao planejamento e implementação de medidas profiláticas exequíveis no contexto social da área;

- à pronta abordagem diagnóstica e terapêutica;

- à avaliação permanente da malária no Brasil, desde uma perspectiva que inclua seus múltiplos determinantes - culturais, ecológicos, econômicos e sociais.

Desse modo, poderão ser alcançados os objetivos:

- minimização da mortalidade;

- redução das perdas sociais e econômicas causadas pela maleita, com redução da morbidade;

- desenvolvimento de estratégias para, no futuro, aprimorar-se as ações de controle.

Em 2015, a Assembleia Mundial de Saúde aprovou a "Estratégia Mundial contra a Malária (2016-2030), para os países endêmicos. A estratégia visa à redução da incidência e mortalidade da doença em até $90 \%$, e a eliminação em pelo 35 países até 2030; além de impedir que a doença reapareça onde está eliminada (42).

A partir da documentação epidemiológica da malária, estão sendo introduzidas inúmeras medidas para seu controle (43), tais como abordagens baseadas em marcadores moleculares (vigilância molecular), visando interpretar, de maneira correta, 
os padrões emergentes no contexto de eliminação (44). Esses marcadores fornecem uma profunda análise dos padrões de fluxo de genes, assim como de linhagens de protistas mantidas ao longo do tempo. Tem havido um interesse crescente na estrutura da população de parasitas da malária e notou-se que os microssatélites são marcadores úteis e abundantes nos genomas do $P$. falciparum e $P$. vivax (45). Entretanto, um problema com relação a esses marcadores pode ser a alta taxa de mutação, além do efeito das estruturas populacionais devido a processos demográficos sobre eles - tais como expansões clonais em áreas de baixa transmissão. Estudos epidemiológicos moleculares devem considerar heterogeneidades temporais nas estruturas populacionais do parasita (44).

Com essas intervenções de controle, tem se identificado uma tendência declinante do número de casos e de mortes pela doença $(46,47)$. Dentre as maneiras de prevenção da doença, destacam-se, nesta seção do artigo, o controle vetorial e o possível desenvolvimento de uma vacina.

\subsection{Controle vetorial}

Com o advento do Dicloro-Difenil-Tricloroetano (DDT), em meados da década de 40, houve grande euforia em relação ao controle da malária, acreditando-se, inclusive, na possibilidade de erradicação da doença. Campanhas patrocinadas pela Organização Mundial da Saúde (OMS) foram levadas a cabo em várias áreas malarígenas do planeta - exceção feita a maior parte do continente africano -, obtendo-se êxito em regiões desenvolvidas, tanto nos países ricos quanto nos mais pobres. Por exemplo, no Brasil a malária foi eliminada das regiões Sul, Sudeste e da maior parte do Nordeste (48).

A despeito das boas possibilidades evocadas com a chegada do DDT, o tempo mostrou que as ações de controle da malária estavam apenas se iniciando, pois não dependia única e exclusivamente do inseticida (48), mas sim de profundas transformações sociais nas localidades acometidas. As comunidades mais pobres e marginalizadas geralmente são mais propensas à malária, já que o ambiente é mais propício aos mosquitos. Além disso, geralmente a pobreza pode reduzir a probabilidade de adoção das medidas profiláticas adequadas como dormir sob uma rede tratada com inseticida, ou uso de mosquiteiros nos dormitórios e, também, das medidas curativas, que buscam o cuidado dos doentes (49).

Em 1970, por ocasião da nova onda de ocupação desordenada da Amazônia, observouse um recrudescimento do número de casos da doença. O Brasil, então, foi dividido em duas regiões, uma na qual a erradicação deveria ser levada a cabo em longo prazo Região Amazônica - e outra em curto prazo - o restante do país(40). Houve mudanças também na abordagem dos métodos de controle da endemia, deslocando-se a ênfase do controle do mosquito para os cuidados com o doente, visando minorar a morbidade e mortalidade associadas à moléstia.

Devido à grande capacidade de os insetos se reproduzirem, é difícil atingir o objetivo de controle vetorial. Além disso, o controle químico pode ser tóxico, causar a mortalidade de outros seres que não o vetor e levar à resistência aos inseticidas. Por isso, têm sido desenvolvidas outras estratégias - dentre elas a técnica do inseto estéril método específico e ambientalmente aceitável (7).

\subsection{Vacinação para malária}

Ao contrário de outras enfermidades infecciosas, não existe vacina para malária. Esforços internacionais vêm sendo intensificados com esse objetivo, havendo esperança de que, brevemente, uma vacina seja licenciada e disponibilizada para países endêmicos (50). 
Nos últimos cinco anos, houve um grande progresso para o desenvolvimento de vacinas para a malária. A primeira tentativa de vacina recombinante dirigida ao Plasmodium falciparum, RTS, S/AS01, encontra-se em testes de Fase III. Enquanto isso, já se trabalha na elaboração de uma segunda-geração de vacina. Nos próximos anos, espera-se que haja grandes estudos para o desenvolvimento de vacinas contra malária placentária, destinadas à prevenção da transmissão da malária, e contra o $P$. vivax $(50,51)$. Existem estudos baseados em um modelo do ciclo de vida do vetor e do protozoário - bem como no funcionamento do sistema imunológico - para prever o impacto de potenciais vacinas para malária em áreas endêmicas e não endêmicas (52). Áreas de baixa transmissão são alvos potenciais para a eliminação da doença $(43,52)$.

Dentre as estratégias de vacinação, uma delas visa à interrupção do desenvolvimento sexual e fertilização do Plasmodium - etapa essencial do ciclo de vida -, bloqueando a transmissão do protista. Entretanto, uma vacina eficaz precisa ter como alvo os diversos estágios de evolução das diferentes formas parasitárias. Tal conhecimento tem sido buscado por meio dos estudos proteômicos (53).

Dentre os problemas para o desenvolvimento de vacinas eficazes, podemos destacar a existência de mais de 5300 proteínas, a expressão de proteínas específicas pelas diferentes fases parasitárias e sexo, e os diferentes mecanismos de resposta imunológica para determinantes antigênicos específicos de cada estágio. Portanto, é necessário identificar as proteínas envolvidas na interação hospedeiro-protozoário para que se possam selecionar os antígenos capazes de induzir resposta imune que eliminará a infecção (54).

Doolan e colaboradores, utilizando a malária como modelo, fizeram uso da técnica de microarrays para elucidar o perfil de anticorpos que se desenvolvem após infecção naturallexperimental ou após a vacinação com organismos atenuados. Microarrays de proteínas foram sondados com soro de grupos que apresentavam estados imune distintos. Cada grupo de doadores apresentou diferentes perfis de anticorpos. A partir dessa técnica, há possibilidade de identificação dos antígenos imunorreativos, o que facilitará o desenvolvimento de vacinas $(45,55)$. Portanto, as estratégias profiláticas serão mais eficazes se orientadas para proteínas próprias expressas em fases específicas do ciclo de vida do Plasmodium (56).

\section{Considerações finais}

A compreensão dos aspectos epidemiológicos da malária - distribuição geográfica, impacto epidemiológico, mecanismo de transmissão, emergência e reemergência, malária resistente e multirresistente no Brasil - são imprescindíveis para o efetivo controle da doença no país. Entretanto, sabemos que conhecer somente não leva às mudanças necessárias para a erradicação e controle de doenças infecciosas em nosso país e no mundo. A educação e a informação são essenciais, mas não suficientes; devem vir acompanhadas de vontade política e recursos financeiros, afim de que possamos sair das metas escritas para a realidade operada. É notório que doenças como a malária têm grande impacto na saúde das pessoas no mundo, contudo, apesar desse cenário, descuidos com a saúde coletiva são claros e recorrentes no Brasil, como podemos ver com a epidemia de dengue e zika (57) e com os surtos de febre amarela de grande magnitude que vêm ocorrendo nos anos de 2017 e 2018, afetando, inclusive, grandes cidades (58).

Precisamos estar atentos, considerando o atual contexto neoliberal, de dominação externa, em associação com o desmantelamento da saúde pública e da ciência no Brasil, que o controle da malária, assim como o de diversas outras enfermidades como a febre amarela, o dengue e as doenças imunopreveníveis, não se transforme em uma quimera, uma utopia inalcançável para a sociedade e para um povo, que, ainda, padece da fome e 
falta de acesso à saúde.

\section{Referências}

1. Martínez-Salazar E, Tobón-Castño A, Blair S. Malaria em humanos por infección natural con Plasmodium knowlesi. Biomédica 2012; 32(Sup 1):121-30.

2. Servonnet A, Rapp C, Delacour H, Bigaillon C, Pilo JE, Mérens A. Plasmodium knowlesi: an emerging species in humans? Med Sante Trop 2012; 22(4):417-21.

3. Brasil. Ministério da Saúde. Secretaria de Vigilância em Saúde. Departamento de Vigilância Epidemiológica. Guia prático de tratamento da malária no Brasil. Brasília: Ministério da Saúde, 2010 [citado 20 de agosto de 2013]. $36 \quad$ p. Disponível em http://bvsms.saude.gov.br/bvs/publicacoes/guia_pratico_malaria.pdf

4. White NJ, Breman JG. Malária. In: Fauci AS, Braunwald E, Kasper DL, et al. Harrison Medicina Interna, Rio de Janeiro, $17^{\mathrm{a}}$ ed, McGraw-Hill, 2008;1280-1294.

5. Ferreira MS. Malária: patologia, fisiopatologia, quadro clínico e diagnóstico. In: Focaccia R. Veronesi: tratado de infectologia. $3^{\mathrm{a}}$ ed. São Paulo: Atheneu, 2005; p. 1615-1624.

6. Raposo CC, Santos JB, dos Santos GM, Gonçalves Eda G, da Silva AR. Plasmodium vivax malaria: related factors to severity in the State of Maranhão, Brazil. Rev Soc Bras Med Trop 2013; 46(1):6772.

7. Tauil PL. Malária: Epidemiologia e Controle. In: Veronesi R, Foccacia R. Tratado de Infectologia. $3^{\mathrm{a}}$ ed. São Paulo; Atheneu, 2005. p. 1625-1628.

8. World Health Organization. WHO guidelines for the treatment of malaria. Geneva $(\mathrm{CH})$ : WHO; 2010 .

9. World Health Organization. World malaria report 2017. Geneva: World Health Organization; 2017. Disponível em: http://www.who.int/malaria/publications/world-malaria-report-2017/en/. Acesso em: 02 de fevereiro de 2018.

10. Brasil, Ministério da Saúde. Secretaria de Vigilância em Saúde. Sistema de Informação de Vigilância Epidemiológica da Malária SIVEP-Malária. Situação epidemiológica da malária na região Amazônica, período de 2015 a 2017. Disponível em: https://public.tableau.com/profile/mal.ria.brasil\#!/vizhome/MiniSivep1517_2018_01_04/casos_notifi cados_2017_regio_Amaznica. Acesso em 02 de fevereiro de 2018.

11. Reiners AAO, Azevedo RCS, Ricci HA, Souza TG. Adesão e reação de usuários ao tratamento da malária: implicações para a educação em saúde. Texto \& Contexto Enferm 2010;19(3):536-544.

12. Souza JM, Couto AARA, Silva EB, Abdon NP, Silva RSU. Malária. In: Queiroz de Leão RN. Doenças Infecciosas e Parasitárias: enfoque amazônico. Belém: Cejup; 1997. p. 38-43.

13. Oliveira-Ferreira J, Lacerda MV, Brasil P, Ladislau JL, Tauil PL, Daniel-Ribeiro CT. Malaria in Brazil: na overview. Malaria Journal 2010; 115(9):1-15.

14. Costa AP, Bressan CS, Pedro RS, Valls-de-Souza R, Silva S, Souza PR de et al. Diagnóstico tardio de malária em área endêmica de dengue na extra-Amazônia Brasileira: experiência recente de uma unidade sentinela no estado do Rio de Janeiro. Rev Soc Bras Med Trop 2010; 43(5): 571-574.

15. Rey L. Malária. Em. Rey L. Parasitologia. 4ª Ed. Rio de Janeiro: Guanabara Koogan; 2008.

16. Lardeux F, Aliaga C, Tejerina R, Torrez L. Comparison of transmission parameters between Anopheles argyritarsis and Anophelespseudopunctipennis in two ecologically different localities of Bolivia. Malar J 2013:13; 12(1):282.

17. Gomes AC, Paula MB, Duarte AMR, Lima MA, Malafronte RS, Mucci LF, et al. Epidemiological and ecological aspects related to malaria in the area of influence of the lake at Porto Primavera dam, in western São Paulo State, Brazil. Rev Inst Med trop S Paulo 2008:50(5):287-295.

18. Scuracchio P, Vieira SD, Dourado DA, Bueno LM, Colella R, Ramos-Sanchez EM, et al. Transfusion-transmitted malária: case report of asymptomatic donor harboring Plasmodium malariae. Rev Inst Med Trop Sao Paulo 2011; 53(1):55-9.

19. Anthony MP, Burrows JN, Duparc S, Moehrle JJ, Wells TN. The global pipeline of new medicines for the control and elimination of malaria. Malar J 2012; 11:316. 
20. Valle D, Clark J. Conservation efforts may increase malaria burden in the Brazilian Amazon. PLoS One 2013; 8(3):e57519.

21. Brasil LW, Rodrigues-Soares F, Santoro AB, Almeida ACG, Kühn A, Ramasawmy R, Lacerda MVG, Monteiro WM, Suarez-Kurtz G. CYP2D6 activity and the risk of recurrence of Plasmodium vivax malaria in the Brazilian Amazon: a prospective cohort study. Malar J 2018; 17(1):57-62.

22. Cohen JM, Smith DL, Cotter C, Ward A, Yamey G, Sabot OJ, et al. Malaria resurgence: a systematic review and assessment of its causes. Malar J 2012; 11:122-138.

23. Lorenz C, Virginio F, Aguiar BS, Suesdek L Chiaravalloti-Neto F. Spatial and temporal epidemiology of malaria in extra-Amazonian regions of Brazil. Malaria Journal 2015 (14):408.

24. Patnaik P, Jere CS, Miller WC, Hoffman IF, Wirima J, Pendame R, et al. Effects of HIV-1 serostatus, HIV-1 RNA concentration, and CD4 cell count on the incidence of malaria infection in a cohort of adults in rural Malawi. J Infect Dis. 2005:15; 192(6):984-91.

25. Cohen C, Karstaedt A, Frean J, Thomas J, Govender N, Prentice E, et al. Increased prevalence of severe malaria in HIV-infected adults in South Africa. Clin Infect Dis. 2005; 41(11):1631-7.

26. Grimwade K, French N, Mbatha DD, Zungu DD, Dedicoat M, Gilks CF. HIV infection as a cofactor for severe falciparum malaria in adults living in a region of unstable malaria transmission in South Africa. AIDS 2004; 18(3):547-54.

27. Van Geertruyden JP, Mulenga M, Mwananyanda L, Chalwe V, Moerman F, Chilengi R, et al. HIV-1 immune suppression and antimalarial treatment outcome in Zambian adults with uncomplicated malaria. J Infect Dis 2006; 194(7):917-25.

28. Kamya MR, Gasasira AF, Yeka A, Bakyaita N, Nsobya SL, Francis D, et al. Effect of HIV-1 infection on antimalarial treatment outcomes in Uganda: a population-based study. J Infect Dis 2006; 193(1):9-15.

29. Mermin J, Lule JR, Ekwaru JP. Association between malaria and CD4 cell count decline among persons with HIV. J Acquir Immune Defic Syndr 2006; 41(1):129-30.

30. Alemu A, Shiferaw Y, Addis Z, Mathewos B, Birhan W. Effect of malaria on HIV/AIDS transmission and progression. Parasit Vectors 2013;6:18.

31. Schneider KA, Kim Y. An Analytical Model for Genetic Hitchhiking in the Evolution of Antimalarial Drug Resistance. Theor Popul Biol 2010; 78(2):93-108.

32. Pohlit AM, Lima RB, Frausin G, Silva LF, Lopes SC, Moraes CB, et al. Amazonian plant natural products: perspectives for discovery of new antimalarial drug leads. Molecules 2013; 18(8):9219-40.

33. Na-Bangchang K, Muhamad P, Ruaengweerayut R, Chaijaroenkul W, Karbwang J. Identification of resistance of Plasmodium falciparum to artesunate-mefloquine combination in an area along the Thai-Myanmar border: integration of clinico-parasitological response, systemic drugexposure, and in vitro parasite sensitivity. Malar J. 2013 12:263-276.

34. Griffing SM, Viana GM, Mixson-Hayden T, Sridaran S, Alam MT, de Oliveira AM, et al. Historical shifts in Brazilian P. falciparum population structure and drug resistance alleles. PLoS One. 2013; 8(3):e58984.

35. Majori G. Combined antimalarial therapy using artemisinin. Parassitologia 2004; 46(1-2):85-7.

36. Siswantoro H, Russell B, Ratcliff A, Prasetyorini B, Chalfein F, Marfurt J, et al. In vivo and in vitro efficacy of chloroquine against Plasmodium malariae and P. ovale in Papua, Indonesia. Antimicrob Agents Chemother 2011; 55(1):197-202.

37. Santos Silva J, Alencar J, Costa JM, Seixas-Lorosa E, Guimarães AÉ. Feeding patterns of mosquitoes (Diptera: Culicidae) in six Brazilian environmental preservation areas. J Vector Ecol 2012; 37(2):342-50.

38. Secretaria de Vigilância em Saúde. Programa Nacional de Prevenção e Controle da Malária PNCM / Ministério da Saúde, Secretaria de Vigilância em Saúde. Brasília: Ministério da Saúde; 2003.

39. Stefani GP, Pastorino AC, Castro APBM, Fomin ABF, Jacob CMA. Repelentes de insetos: recomendações para uso em crianças. Rev Paul Pediatr 2009; 27(1):81-9

40. Secretaria de Vigilância em Saúde. Diretoria Técnica de Gestão. Guia para profissionais de saúde sobre prevenção da malária em viajantes / Ministério da Saúde, Secretaria de Vigilância em Saúde, Diretoria Técnica de Gestão. Brasília: Ministério da Saúde; 2008.

41. World Health Organization. Goals, targets, policies and strategies for malaria control and 
elimination: Genebra; 2011.

42. Organização Pan-Americana da Saúde/ Organização Mundial Da Saúde. Malária. Brasil, Abr. 2016. Disponível http://www.paho.org/bra/index.php?option=com_content\&view=article\&id=5287:malaria$2 \&$ Itemid=875. Acesso em: 05 de fevereiro de 2018.

43. Chenet SM, Schneider KA, Villegas L, Escalante AA. Local population structure of Plasmodium: impact on malaria control and elimination. Malaria J 2012; 11:412-424.

44. Liu C, Zwiebel LJ. Molecular Characterization of Larval Peripheral Thermosensory Responses of the MalariaVector Mosquito Anopheles gambiae. PLoS One. 2013; 8(8):e72595.

45. Siqueira-Batista R, Gomes AP, Mendonça EG, Vitorino RR, Azevedo SFM, Freitas RB, et al. Malária por Plasmodium falciparum: estudos proteômicos. Rev Bras Ter Intensiva. 2012; 24(4):394-400.

46. Masaninga F, Chanda E, Chanda-Kapata P, Hamainza B, Masendu HT, Kamuliwo M, et al. Review of the malaria epidemiology and trends in Zambia. Asian Pac J Trop Biomed 2013; 3(2):89-94.

47. Chiyaka C, Tatem AJ, Cohen JM, Gething PW, Johnston G, Gosling R, et al. Infectious disease. The stability of malaria elimination. Science 2013; 339(6122):909-10.

48. Ramirez JL, Garver LS, Dimopoulos G. Challenges and Approaches for Mosquito Targeted Malaria Control. Curr Mol Med 2009; 9(2): 116-130.

49. Ricci F. Social Implications of Malaria and Their Relationships with Poverty. Mediterr J Hematol Infect Dis 2012; 4; Open Journal System.

50. Riley EM, Stewart VA. Immune mechanisms in malaria: new insights in vaccine development. Nat Med 2013; 19(2):168-78.

51. Heppner DG. The malaria vaccine - status quo 2013. Travel Med Infec Dis 2012; 11(1): 2-7.

52. Wenger EA, Eckhoff PA. A mathematical model of the impact of present anfd future malaria vaccines. Malaria Journal, 2013; 12: 126-138.

53. Khan SM, Franke-Fayard B, Mair GR, Lasonder E, Janse CJ, Mann M, et al. Proteome analysis of separated male and female gametocytes reveals novel sex-specific Plasmodium biology. Cell 2005; 121(5):675-87.

54. R, Ivone Castro G, María del Carmen Rodríguez. Análisis proteómico de Plasmodium, el agente causal de la malaria. Salud pública Méx 2009, 51(3): 395-s402.

55. Doolan DL, Mu Y, Unal B, Sundaresh S, Hirst S, Valdez C, et al. Profiling humoral immune responses to $P$. falciparum infection with protein microarrays. Proteomics 2008; 8(22):4680-94.

56. Ramaprasad A, Pain A, Ravasi T. Defining the protein interaction network of human malaria parasite Plasmodium falciparum. Genomics 2012; 99(2):69-75.

57. Brasil. Ministério da Saúde. Secretaria de Vigilância em Saúde. Departamento de Vigilância Epidemiológica. Boletim Epidemiológico. Monitoramento dos casos de dengue, febre de chikungunya e febre pelo vírus zika até a semana epidemiológica 19, 2017. p. 1-10.

58. Minas Gerais. Secretaria de Estado de Saúde de Minas Gerais. Subsecretaria de Vigilância e Proteção à Saúde. Superintendência de Vigilância Epidemiológica, Ambiental e Saúde do Trabalhador. Boletim Epidemiológico. Febre Amarela Silvestre em Minas Gerais. 6 de fevereiro de 2018. p. 1-9. 